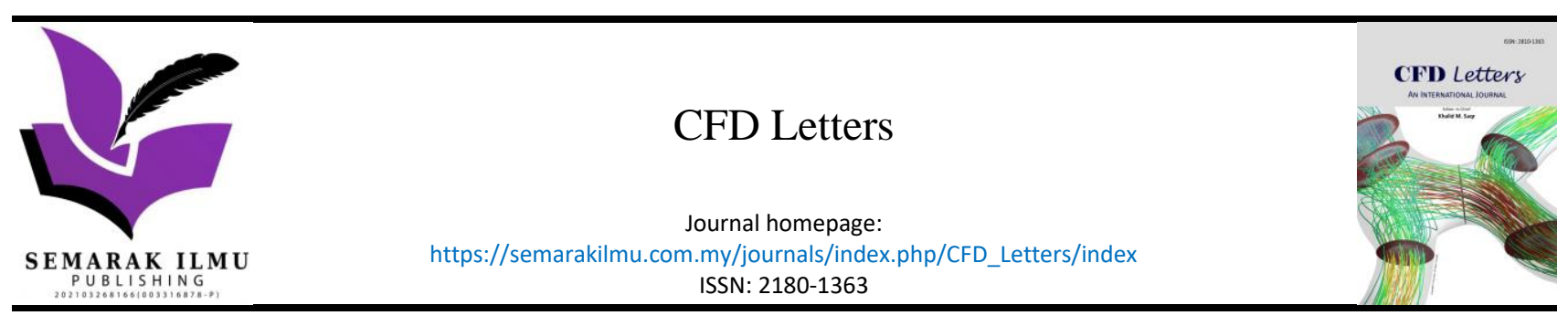

\title{
Multiphase CFD Investigation on Convective Heat Transfer Enhancement for Turbulent Flow of Water- $\mathrm{Al}_{2} \mathrm{O}_{3}$ Nanofluid
}

\author{
Suaib Al Mahmud ${ }^{1}$, Ahmad Faris Ismail ${ }^{1, *}$ \\ Department of Mechanical Engineering, Kulliyyah of Engineering, International Islamic University Malaysia, 53100 Gombak, Kuala Lumpur, \\ Malaysia
}

\section{ARTICLE INFO}

\section{Article history:}

Received 14 August 2021

Received in revised form 10 September 2021

Accepted 11 September 2021

Available online 31 October 2021

\section{ABSTRACT}

Because of extraordinary heat transfer capability, nanofluids have become a potential interest in engineering sectors. Despite being a multiphase fluid, nanofluids were treated as single phase fluids in many previous studies and comparison between single and two phase models was drawn. Examining nanofluids capability to augment heat transfer is one of the keys to utilize them properly in the field of thermofluids. However, the optimal multiphase model to simulate nanofluids heat transfer enhancement is yet to be found out. In this study, the method of computational fluid dynamics has been used to simulate flow of water-Al2O3 nanofluid in a circular pipe in the purpose of identifying the best multiphase model to simulate heat transfer enhancement of nanofluids. Two multiphase models have been taken into account: Volume of Fluid and Mixture model. Three different volume fractions of nanoparticles in nanofluid have been tested for each of these models such as $1 \%, 4 \%$ and $6 \%$ for highly turbulent flows where Reynolds number was ranged between 20000 to 80000 . The standard $k-\varepsilon$ turbulence model has been employed to model the flow of nanofluid with the mentioned multiphase models in the present study. The results have been carried out in forms of correlation between Re and $\mathrm{Nu}$ and have been compared with existing experimental results. The results showed that the heat transfer enhancement of nanofluid is mostly dominated by concentration of nanoparticles present in the fluid and suggested that Mixture model is suitable for predicting convective heat transfer enhancement of nanofluid for cases with high particle concentration though the necessity of further experimental study in some scopes has been detected.
Keywords:

Nanofluid; forced convection; multiphase; heat transfer

\section{Introduction}

A range of engineering instruments and systems operate depending on the mechanism of heat transfer and their basic working principle includes a fluid to transfer the heat from one part to another whose efficiency depends upon the heat transfer capability of the working fluid. The time was 1873 and it was the revolutionary idea of Maxwell to add solid particles to Heat Transfer Fluids (HTF) to increase their thermal conductivity [1]. While the addition of micrometer and millimeter sized solid particles was causing issues such as clogging, increased pressure drop and pipe erosion,

\footnotetext{
* Corresponding author.

E-mail address: faris@iium.edu.my (Ahmad Faris Ismail)
}

https://doi.org/10.37934/cfdl.13.10.1124 
some light was shed on this concept when S.U.S Choi and J. Eastman introduced nanoscale metallic particles and carbon nanotubes as nanoparticles where they conducted experiments with variety of fluids and found out the results to be satisfactory [2]. Since then, nanofluids are known for their improved heat transfer characteristics and have successfully proved their importance in the industrial atmosphere in terms of transferring heats. The key variable that controls the thermophysical properties and heat transfer capability of nanofluids is the nanoparticle volume concentration [3]. Nanoparticles are spherical solid particles those have a diameter of less than $100 \mathrm{~nm}$ such as $\mathrm{Al}_{2} \mathrm{O}_{3}$, $\mathrm{CuO}, \mathrm{TiO}_{2}, \mathrm{Cu}, \mathrm{Fe}$ and carbon-based material such as carbon nanotubes (CNTs). Over past few years many studies have been conducted on the improved heat transfer behavior and on the thermophysical properties of nanofluid. While this is quite apparent that nanofluid is a multiphase fluid because of the presence of two different phases such as base fluid which is a liquid and nanoparticles which are solid, several studies have been carried out on nanofluids assuming them as single phase fluid. Even in some of the recent works, such as where the thermal and hydrodynamic behavior were examined using 2D CFD simulation, single phase model was depended upon [4]. Though in some cases these assumptions were proved convenient, but they caused several errors too.

A numerical study on laminar convective heat transfer of nanofluids in a circular tube under constant wall temperature was carried out using CFD approach where both single and two phase models were employed. The results displayed comparatively stronger cooperation of two phase model with experimental results than single phase model [5]. A different opinion was made when Saghir et al., [6] carried out an experiment with the flow of nanofluids in a square cavity and compared the result of single and two phase models with experimental results. Their conclusion suggested that though multiphase model provides deep comprehension on the liquid and solid phase mixture but the single phase model predicts the heat transfer more accurately. This finding aligns with the outcome of another numerical investigation of single and multiphase model conducted by Esfandiary et al., [7]. The simplicity of single phase model was acknowledged by Kakaç and Pramuanjaroenkij [8]. But taking slip velocity between nanoparticles and the base fluid they suggested that that the multiphase model provides a better view of nanofluids flow field.

In another recent research on forced convection of nanofluid, single and two phase models were examined. It was seen that two phase model result seems to be more convincing when compared to single phase when compared to experimental data [9]. Another contemporary study picked up that two phase model is more convincing in terms of predicting heat transfer though with the increase in particle concentration the two phase model seem to overestimate the heat transfer enhancement [10]. In a more recent study where single and multiphase models were employed for nanofluids convection at the entrance of a uniformly heated tube. It was picked up that two phase models tend to predict heat transfer parameters better than single phase model [11]. After that Safaei et al., [12] conducted a review of latest works on nanofluids mathematical modelling for simulation. Though they concluded that realistic results can be achieved by using temperature-dependent thermophysical properties of nanofluid in homogeneous model but vouched for better accuracy of two phase models. Subsequently, comparison of heat and mass transfer between single and two phase models was drawn via numerical study for nanofluid liquid film which was flowing downward a vertical channel. It was deduced that two phase model was more practical as it accounts for thermophoresis and Brownian effects [13]. Latterly, Ambreen et al., [14] carried out very comprehensive research on single phase and the multiphase models where they noticed that at low Reynolds number, single and multiphase models can precisely predict the thermal fields of diluted nanofluids but at denser concentration and higher Reynold number the results are overestimated. In 
all these investigations, the main focus was to make comparison between single and multiphase models.

Now, there are two approaches of looking into a multiphase fluid flow. The first one is called the Euler-Langrange Approach which treats the fluid phase as a continuum by solving the momentum equations, but a large number of particles are tracked through the calculated flow field for solving the dispersed phase. It allows the dispersed phase to interact with the fluid phase by exchanging momentum, mass, and energy. In this model, there is a presumption. That is, the dispersed second phase takes up a small volume fraction, despite the fact that high mass loading is acceptable. During the calculation of fluid phase, the particle or droplet trajectories are computed individually at specified intervals.

The seccond one is known as Euler-Euler approach where the distinct phases are mathematically handled as interpenetrating continuum considering idea of phasic volume fraction since the volume of a phase cannot be filled by other phases. These volume fractions are believed to be continuous space and time functions with a mathematical total of one. Conservation equations for each phase are generated to obtain a set of equations with a similar structure for all phases. These equations are solved by supplying constitutive relations based on observations. There are three different EulerEuler multiphase models available: the volume of fluid (VOF) model, the mixture model, and the Eulerian model among which Mixture model and VOF model has been used to simulate the flow of nanofluid in this study.

The VOF model is a surface tracking technique employed on a Eulerian mesh which is fixed. It has been designed for two or more fluids those are unable to form a homogenous mixture where point of interest is the interface betwen the fluids considered. The fluids in this model share a single set of momentum equations, and each fluids volume fraction in each computational cell is monitored across the domain.

For treating two or more phases (fluid or particulate) Mixture model is designed. The phases are considered as interpenetrating continua in the Euler-Euler technique. Mixture momentum equation is solved by the mixture model, taking into consideration the relative velocities of the phases, and by prescription of it the model describes the dispersed phases.

A few researches on above mentioned multiphase models were also conducted. Hanafizadeh et al., [15] examined and compared the multiphase models in terms of predicting heat transfer and found out that at low Reynolds number and developing region of the flow, Mixture model performs better heat transfer coefficient prediction than other models. The study of Lotfi et al., [9] on numerical study of forced convective heat transfer of nanofluids found out that two phase Eulerian model underestimates the Nusselt number. He also concluded mixture model to be more convincing. Meanwhile, Davarnejad and Jamshidzadeh [16] observed that among three multiphase models (VOF, Eulerian and Mixture) VOF and Mixture model predicted friction factor and Nusselt number dramatically.

This is quite apparent that, though numerous researches have been conducted for comparing single and multiphase models, the domain of multiphase models remained heavily understudied. The conducted researches on multiphase models are limited to their particular focuses. Though the scale tipped a little bit on the side of mixture model, VOF model also holds a fair share of its accuracy. This leads to an absolute confusion regarding the best multiphase model for simulating nanofluids heat transfer. In addition, the information on heat transfer behavior of nanofluids for different nanoparticle concentrations is hazy. Which is why examining enhancement of convective heat transfer for turbulent flow of nanofluid with variable particle concentration using multiphase models remains as a vital necessity. Therefore, the present study aims to investigate the behavior of nanofluids convective heat transfer enhancement employing Mixture and VOF models by varying 
particle concentration and Reynolds number. In addition, it intends to find out the most effective multiphase model to simulate nanofluids heat transfer enhancement by comparing the multiphase model results with existing experimental data.

\section{Governing Equations}

There are three principal equations associated with multiphase flows which have been derived from the basic concepts of fluid dynamics. First one is the continuity equation that talks about the conservation of mass. The second one is momentum equation talking about the conservation of momentum. Last one is the energy equation that represents the conservation of energy in a fluid element. All these equations are applicable to the flow of nanofluids.

\subsection{Continuity Equation}

According to this equation, the rate of mass entering the system exactly equals the rate of mass exiting the system plus the aggregation of mass in the system. The continuity equation for nanoparticles and base fluid can be represented as [5]:

$\frac{\partial}{\partial t} \cdot\left(\varphi_{\alpha} \rho_{\alpha}\right)+\nabla \cdot\left(\varphi_{\alpha} \rho_{\alpha} U_{\alpha}-\Gamma_{\alpha} \nabla \varphi_{\alpha}\right)=0$

Here, $\varphi, \rho, U$ and $\Gamma$ stand for volume fraction, density, interstitial velocity vector and dispersion coefficient. The subscript $\alpha$ represents phase index.

\subsection{Momentum Equation}

The momentum equation which is also called Navier-Stokes equation refers to Newton's Second Law relating the rate of momentum change to the number total of forces that act on a component of liquid. The differential form of this equation is as following [5]:

$\frac{\partial}{\partial t} \cdot\left(\varphi_{\alpha} \rho_{\alpha} U_{\alpha}\right)+\left\{\varphi_{\alpha}\left[\rho_{\alpha} U_{\alpha} U_{\alpha^{-}} \mu_{e \alpha}\left(\nabla U_{\alpha}+\left(\nabla U_{\alpha}\right)^{T}\right)\right]\right\}=\varphi_{\alpha}\left(B_{\alpha^{-}} \nabla P\right)+F_{\alpha}$

$T, B, \nabla P$ and $F$ indicate temperature, body force, pressure drop and interphase force respectively.

\subsection{Energy Equation}

The last equation is about the conservation of energy which states that energy cannot be created or destroyed and it only changes from one form to another. The final amount of energy in a system is same as the previous amount of it but just in a different form. In an equational manner it is represented as [5]:

$\frac{\partial}{\partial t} \cdot\left(\varphi_{\alpha} \rho_{\alpha} U_{\alpha}\right)+\nabla \cdot\left[\varphi_{\alpha}\left(\rho_{\alpha} U_{\alpha} h_{\alpha^{-}} k_{\alpha} \nabla T_{\alpha}\right)\right]=\sum_{\beta=1}^{N}\left(\Gamma_{\alpha \beta}+h_{\beta S^{-}} \Gamma_{\beta \alpha} h_{\alpha s}\right)+Q_{\alpha}+S_{\alpha}$

In this equation, enthalpy, thermal conductivity, interphase heat transfer and external heat source in energy equation are denoted by $h, \mathrm{k}, Q$ and $S$, whereas the subscripts $\beta$ and s indicate phase index and solid particles. 


\section{Geometry and Meshing}

For this simulation the geometry considered is a simple pipe as represented in Figure 1, which is horizontally placed with respect to the ground that will have a turbulent flow of nanofluid. The geometry has been obtained from benchmark research paper Bianco et al., [17].

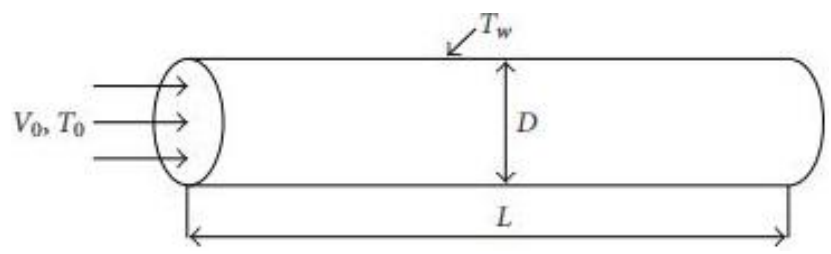

Fig. 1. Geometry for numerical simulation

Geometry specifications are mentioned in Table 1 as following:

Table 1

Geometry specifications

\begin{tabular}{ll}
\hline Specification & Magnitude \\
\hline Material & Aluminium \\
Diameter $(\mathrm{mm})$ & 10 \\
Length $(\mathrm{mm})$ & 1000 \\
Heat transfer flow area $\left(\mathrm{mm}^{2}\right)$ & 31416 \\
Cross sectional area $\left(\mathrm{mm}^{2}\right)$ & 78.54 \\
\hline
\end{tabular}

Meshing is one of the most crucial part of CFD simulation. The accuracy of the analysis hugely depends on meshing. For statistical reliability and higher precision high density meshing is preferred. However, creating a mesh denser than necessary has a lot of side effects also including faulty results. Considering these factors orthogonal mesh was created. Near to the walls the mesh was created to be comparatively denser than the axis of the pipe according to the calculated first cell thickness. Figure 2 and Figure 3 represent the mesh created on the geometry for simulation and the Fluent grid close up respectively.

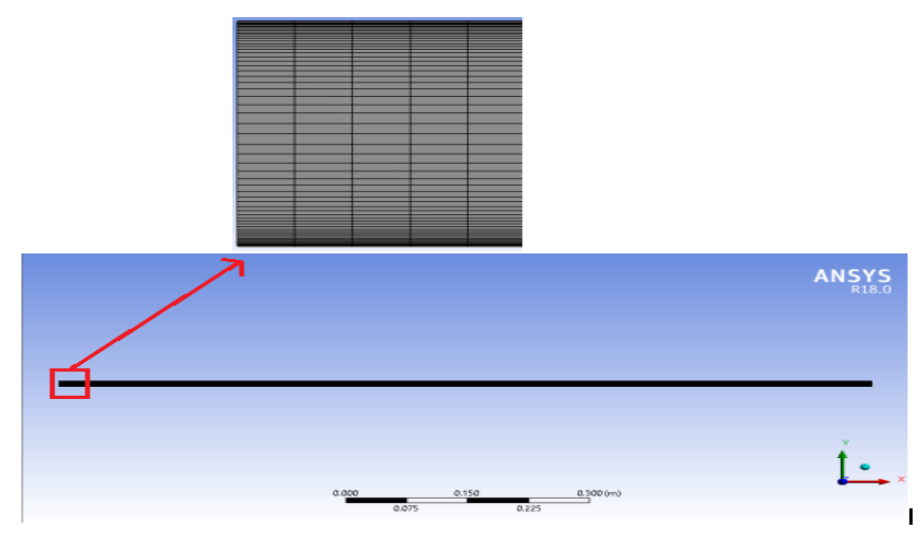

Fig. 2. Created mesh on 2D pipe geometry 


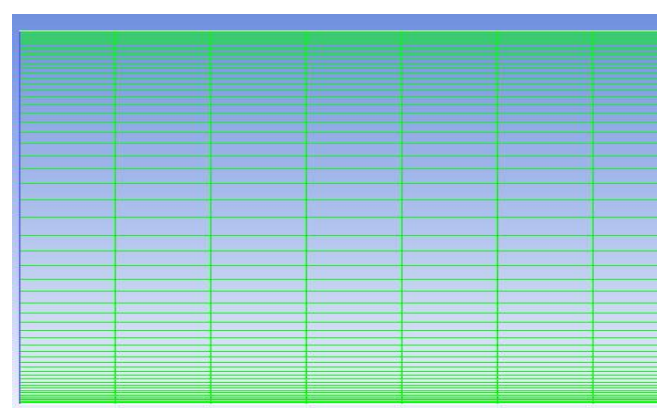

Fig. 3. Grid for simulation in Fluent

\section{Turbulence Modelling}

In this study the standard $k-\varepsilon$ model has been used for simulation of the turbulence flow of water$\mathrm{Al}_{2} \mathrm{O}_{3}$ nanofluid. This model takes turbulent kinetic energy, $k$ and its dissipation rate, $\varepsilon$ into account and they are described as [18]

$$
\begin{aligned}
& \nabla \cdot\left(\rho_{e f f} k U\right)=\nabla \cdot\left[\left(\frac{\mu_{t}}{\sigma_{k}}\right) \nabla(k)\right]+G_{k}-\rho_{e f f} \varepsilon \\
& \nabla \cdot\left(\rho_{e f f} \varepsilon U\right)=\nabla \cdot\left[\frac{\mu_{t}}{\sigma_{\varepsilon}} \nabla \varepsilon\right]+\frac{\varepsilon}{k}\left(C_{1 \varepsilon} G_{k}-C_{2 \varepsilon} \rho_{e f f} \varepsilon\right) \\
& G_{k}=\mu_{t}\left(\nabla U+(\nabla U)^{T}\right) \\
& \mu_{t}=\rho_{e f f} C_{\mu} \frac{k^{2}}{\varepsilon}
\end{aligned}
$$

where, $\mu$ represents viscosity. $C_{1}$ and $C_{2}$ are coefficients in approximated turbulent transport equation. In addition, the subscript eff and $t$ indicate effective and turbulent.

\section{Boundary Conditions}

The boundary conditions for investigation of convective heat transfer for turbulent flow of nanofluid have been obtained from the benchmark paper. The properties of fluid and particles were taken from there as well. In addition, the necessary thermophysical properties of the nanofluid for analysis and simulation were calculated using the formulas provided in the benchmark paper [17]. Table 2 represents the imposed boundary conditions on the geometry for the flow.

Table 2

Boundary conditions used for the simulation

\begin{tabular}{lll}
\hline Zone & Nature & Condition imposed \\
\hline Inlet & Velocity inlet & - Uniform velocity, $\mathrm{V}_{\mathrm{o}}$ \\
& & - Uniform temperature, $\mathrm{T}_{\mathrm{o}}=293 \mathrm{~K}$ \\
Outlet & Pressure outlet & $\begin{array}{l}\text { Gauge pressure }=0 \mathrm{pa} \\
\text { Walls }\end{array}$ \\
& - & - No slip conditions \\
& & - Constant temperature $\mathrm{T}_{\mathrm{w}}=350 \mathrm{~K}$ \\
\hline
\end{tabular}




\section{Mesh Independence Study (MIS)}

Mesh independence test is a crucial step towards CFD simulation. As this is known that the accuracy of the results of CFD simulation depends on how good the mesh is, the optimal mesh has to be designed in a way that output parameter does not change by significant amount when the mesh is manipulated.

For this simulation the meshing was done by creating two different edge sizing, one along the vertical edges and one along the horizontal edges. The mesh independence test has been done by setting outlet average temperature as output parameter and then by varying the number of divisions at the inlet. This is quite apparent that when the number of divisions are changed the nodes, elements and cell numbers vary. Face meshing was done in order to achieve structured mesh. To make the mesh denser near to the walls according to the calculated first cell thickness, biasing method was used.

Five different mesh types were considered where the number of divisions at the inlet were varied from 10 to 50 divisions as presented in Table 3 and for each case outlet average temperature was recorded while keeping an eye towards the convergence iteration. At the same time, the difference between two consecutive iterations was calculated. Figure 4 shows the variation of pipe outlet temperature as element numbers increase due to increase in division.

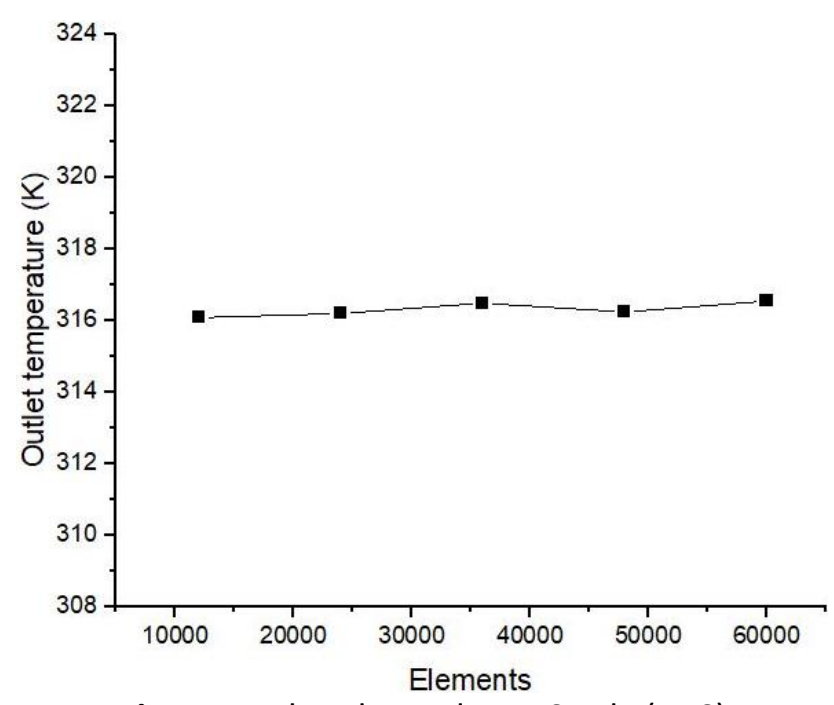

Fig. 4. Mesh Independence Study (MIS)

As seen on Table 3, the difference between mesh type 1 and mesh type 2 was comparatively higher. The difference went down for mesh type 3 and got further reduced by a good amount in mesh type 4. After that it showed a small rise in mesh type 5 . This is noticeable that the differences between outlet temperatures for the mesh types were very insignificant, specially starting from mesh type 3 . It can be observed that, the iteration for convergence was the highest in mesh type 1 . After that it suddenly dropped remarkably and started showing decent increase as divisions were increasing. Considering the differences in outlet temperature and the fact that higher iteration for convergence increases computational time, mesh type 4 was considered as the optimal mesh and all the simulations were run using this mesh type which contains 49241 nodes and 48000 elements. For the optimal mesh, the skewness and mesh quality were tested. The maximum and average skewness for this mesh was $1.3253 \times 10^{-10}$ and $1.3061 \times 10^{-10}$ respectively with an aspect ratio of 3.54781 which are within limits [19]. The orthogonal quality of the mesh is 1.00 which indicates the best orthogonal meshing. 
Table 3

Mesh Independence Study (MIS)

\begin{tabular}{lllllll}
\hline $\begin{array}{l}\text { Mesh } \\
\text { Type }\end{array}$ & Divisions & Nodes & Elements & $\begin{array}{l}\text { Iteration for } \\
\text { Convergence }\end{array}$ & $\begin{array}{l}\text { Outlet average } \\
\text { temperature (K) }\end{array}$ & $\begin{array}{l}\text { Difference } \\
(\%)\end{array}$ \\
\hline 1 & 10 & 13211 & 12000 & 474 & 316.08 & - \\
2 & 20 & 25221 & 24000 & 366 & 316.20 & 0.1267 \\
3 & 30 & 37231 & 36000 & 360 & 316.49 & 0.0379 \\
4 & 40 & 49241 & 48000 & 380 & 316.24 & 0.0032 \\
5 & 50 & 61252 & 60000 & 393 & 316.55 & 0.0727 \\
\hline
\end{tabular}

\section{Validation}

The multiphase models were validated by comparing with the experimental result of Pak and Cho [20] which has also been presented in benchmark research paper Bianco et al., [17]. The same boundary conditions and turbulence model as the benchmark paper such as specified inlet and wall temperature and standard $k-\varepsilon$ turbulence model were used to carry out the result. The validation has been done by examining the change in Nusselt number with Reynolds number ranging from 20000 to 80000 for $4 \%$ particle concentration. Reynolds number and Nusselt number has been calculated using following equations:

$R e=\frac{\rho V D}{\mu}$

$N u=\frac{\mathrm{h} D}{\mathrm{k}}$

Here $V$, h and $D$ express flow velocity at fully developed region, heat flux and pipe diameter. The graphs in Figure 5 represents the comparison between simulated and experimental Re vs $\mathrm{Nu}$ for Mixture model and VOF model respectively for particle concentration of $4 \%$. Both model show very close results. For both cases the error from experimental result ranges from 11 to 15 percent. The average error shown by these two models for 4 different Reynolds numbers are $12.3674 \%$ and $12.3799 \%$ for Mixture and VOF model respectively.

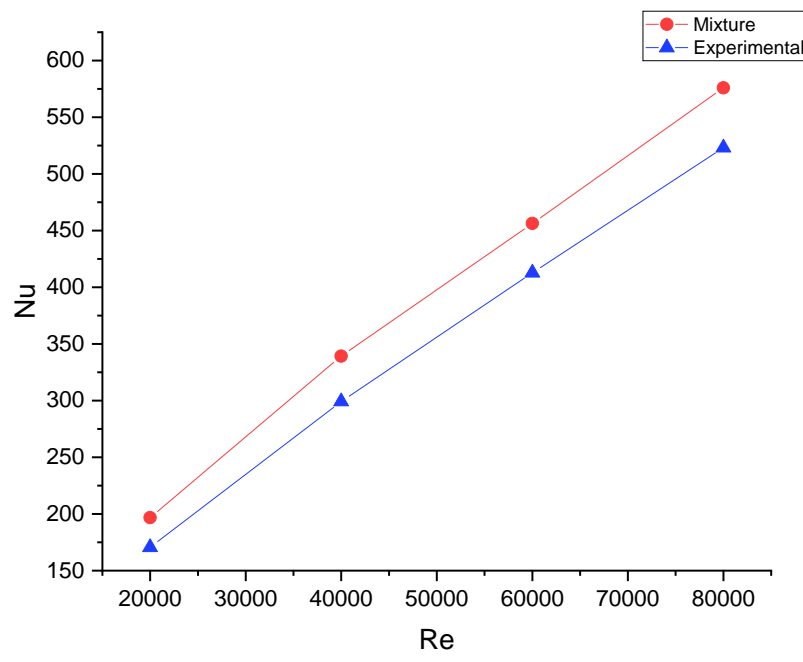

(a)

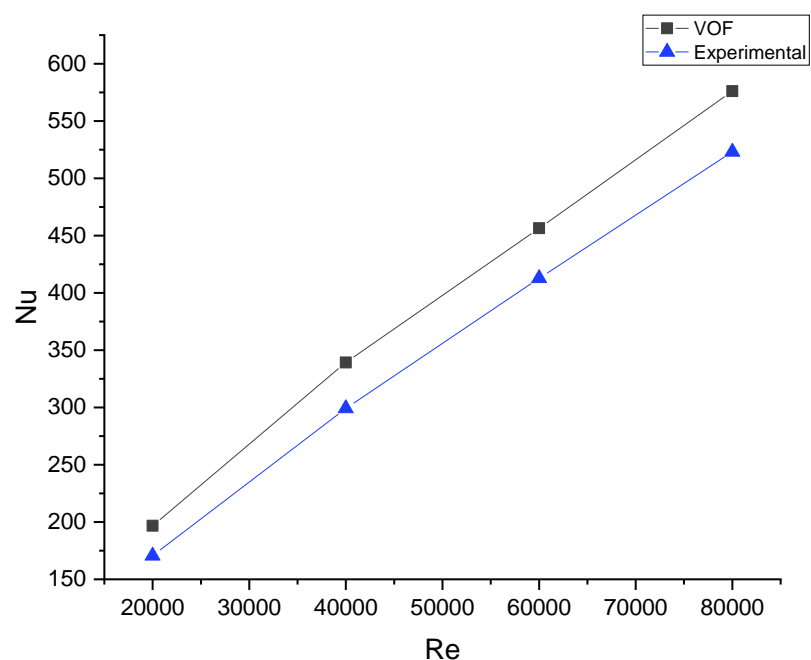

(b)

Fig. 5. Re vs Nu for particle concentration of $4 \%$ 


\section{Results and Discussion}

Three different concentrations $(1 \%, 4 \%$ and $6 \%)$ have been tested using both models by calculating values of $\mathrm{Nu}$ against variable $\mathrm{Re}$. The obtained result via simulation has been compared with the experimental result of Pak and Cho [20] which has also been presented in the benchmark paper. The graphs in Figure 6, Figure 7 and Figure 8 represent Re vs $\mathrm{Nu}$ for $1 \%, 4 \%$ and $6 \%$ concentrations and the variation from the experimental result respectively.

For particle concentration of 1 percent as Re goes from 20000 to 80000 , Nu changes from 104.3131 to 326.4327 for mixture model and from 104.3268 to 326.4359 for VOF model. The average variation from experimental result for mixture model is $29.1955 \%$ and for VOF model is $29.1929 \%$.

As nanoparticle concentration increases by $3 \%$ the pattern of Re vs Nu remains similar but this time both model predicts higher Nu for the same range of Re. Both models depict the same pattern of results while keeping a very small difference between each other. In fact in some cases the results for two models are so close that the difference can be counted as negligible and for a very few cases, specially at lower Re they yielded the same result. As Re goes from 20000 from 80000 the $\mathrm{Nu}$ increases from 196.77 to 575.99 for Mixture model and from 196.77 to 576.13 for VOF model. The results provided by Mixture and VOF model varies from the experimental result by $11 \%$ to $15 \%$. The average difference from experimental result shown by Mixture and VOF models for 4 percent concentration is $12.3674 \%$ and $12.3799 \%$ respectively.

For the highest amount of particle concentration in this study (6\%), Nu ranges between 256.9398 and 721.4352 according to Mixture model and between 256.9616 and 721.9857 according to VOF model as Re ranges between 20000 and 80000 . The difference from experimental result ranges from $25 \%$ to $29 \%$. To be more precise, in average Mixture model holds a variation of $28.6621 \%$ and VOF model holds a variation of $28.6898 \%$ from the experimental result. So, this can be derrived from the results that, for a given Re the heat transfer capability of nanofluids increases with the increase in particle concentration.

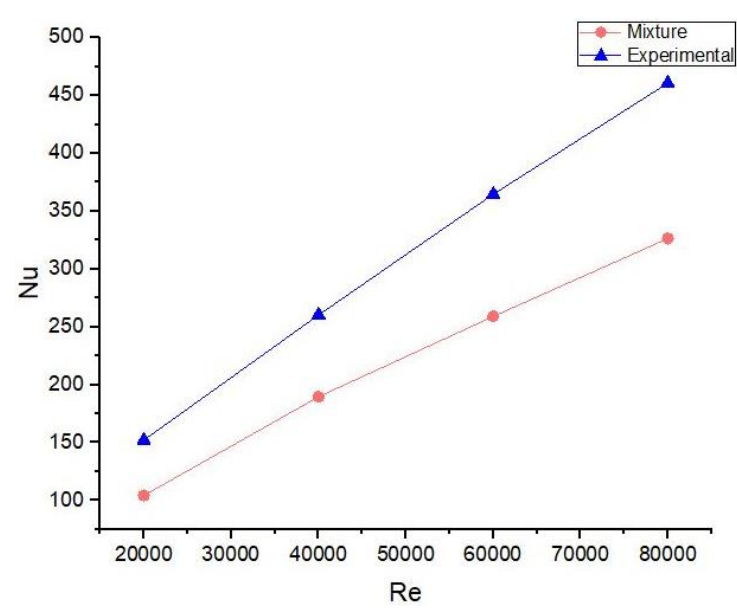

(a)

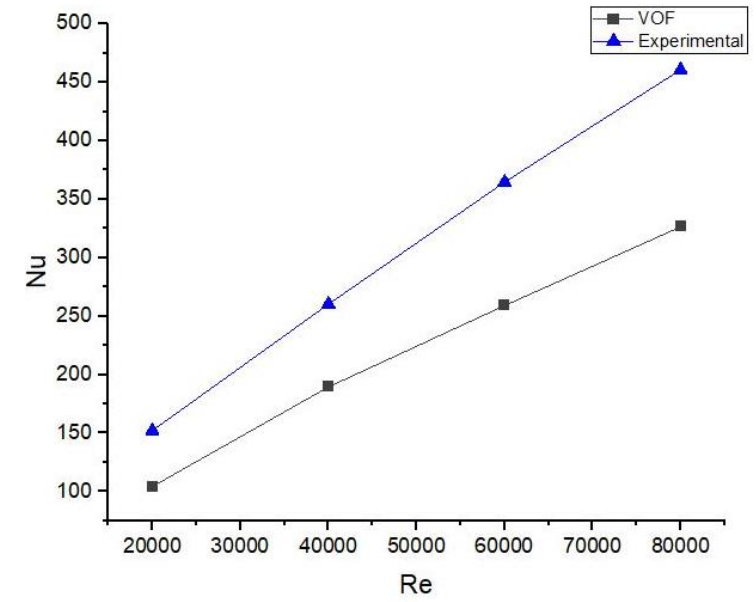

(b)

Fig. 6. Nusselt number with respect to Reynolds number for particle concentration of $1 \%$ 


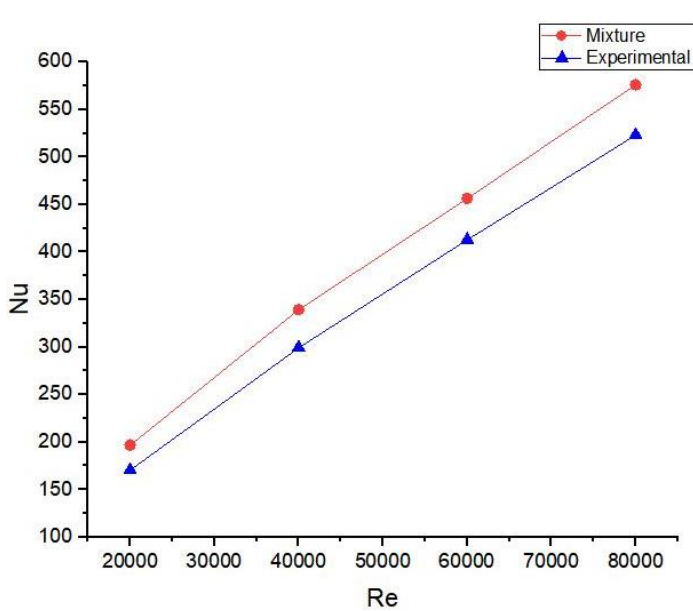

(a)

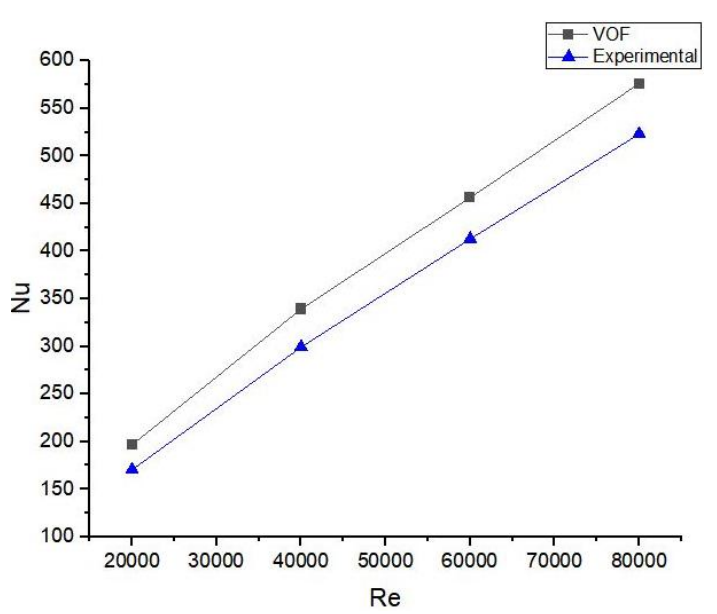

(b)

Fig. 7. Nusselt number with respect to Reynolds number for particle concentration of $4 \%$

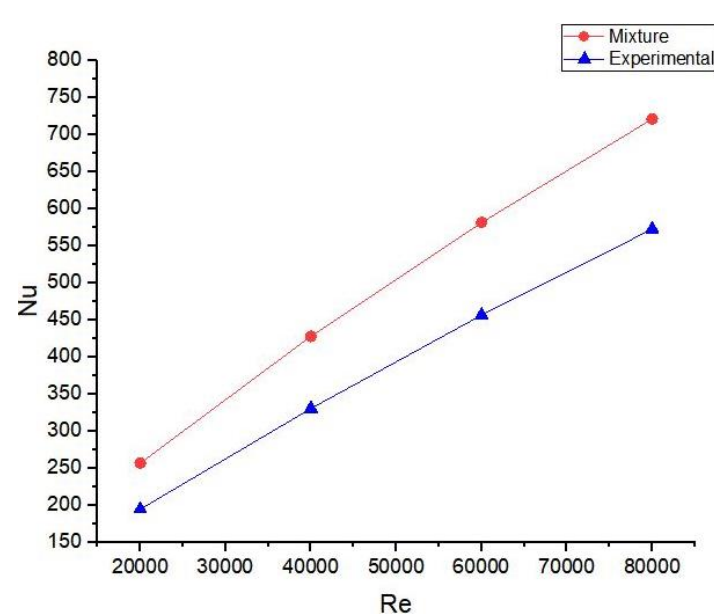

(a)

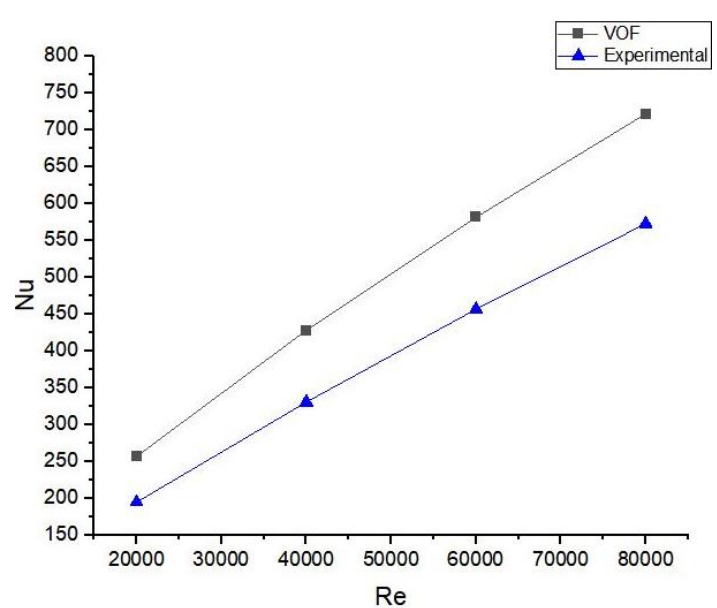

(b)

Fig. 8. Nusselt number with respect to Reynolds number for particle concentration of $6 \%$

For visualizing the amount of difference from experimental data for each multiphase model, a comparison is shown in Table 4 . In the table, the difference range presented refers to the deviation of numerical simulation results from the experimental result as Re moves to 80000 from 20000 . The average difference for each particle concentration has been obtained by calculating the mean value of differences for each Re that has been presented in the graphs. The difference between the results of two models were observed to be very small. In fact the difference between the Nu prediction of these two models are around $0.02 \%$. Still considering this amount of difference it is observed that for particle concentration of $1 \%$, VOF model is more convincing in the prediction of Nu while for particle concentration of $4 \%$ and $6 \%$ mixture model performs better. So, this can be said that for lower concentrations VOF model is more accurate and for higher concentration Mixture model is more convincing for the prediction of convective heat transfer enhancement.

Table 4

Difference between simulated and experimental result

\begin{tabular}{lllll}
\hline Particle concentration(\%) & \multicolumn{2}{l}{ Difference range (\%) } & \multicolumn{2}{l}{ Average difference(\%) } \\
& Mixture model & VOF model & Mixture model & VOF model \\
\hline 1 & $27-29.6$ & $27-29.6$ & 29.1955 & 29.1929 \\
4 & $11-15$ & $11-15$ & 12.3674 & 12.3799 \\
6 & $25-29$ & $25-29$ & 28.6621 & 28.6898 \\
\hline
\end{tabular}




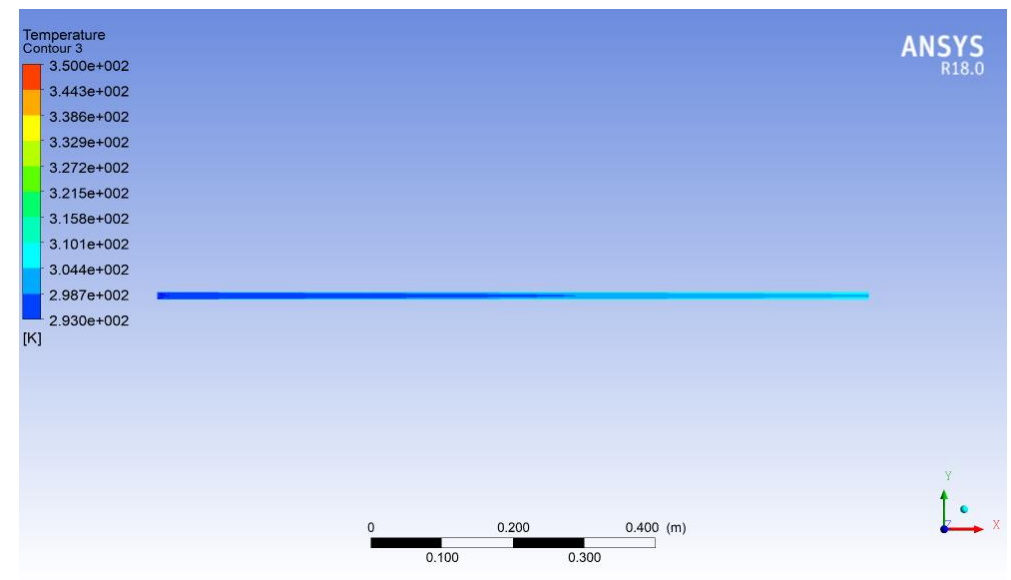

(a)

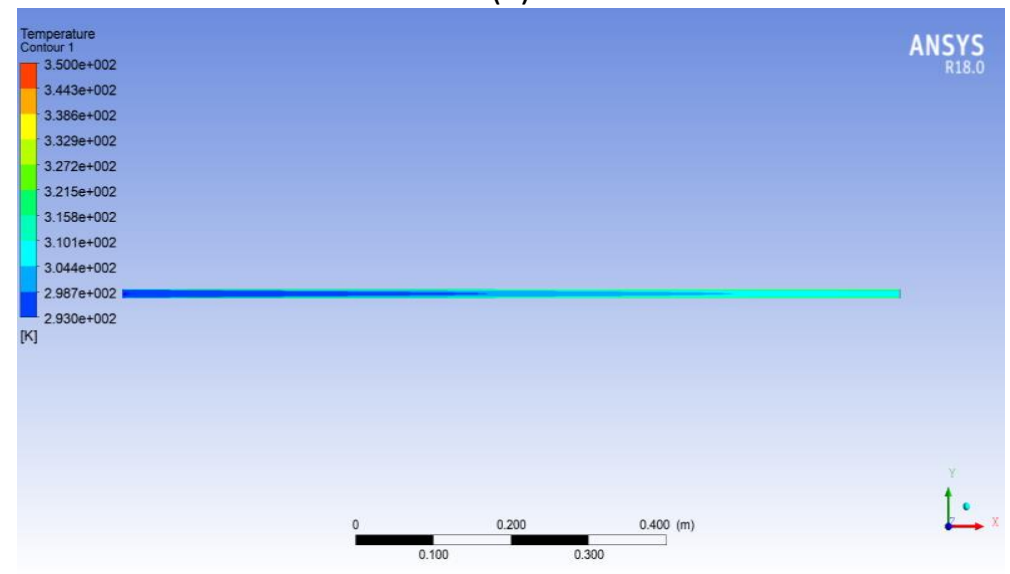

(b)

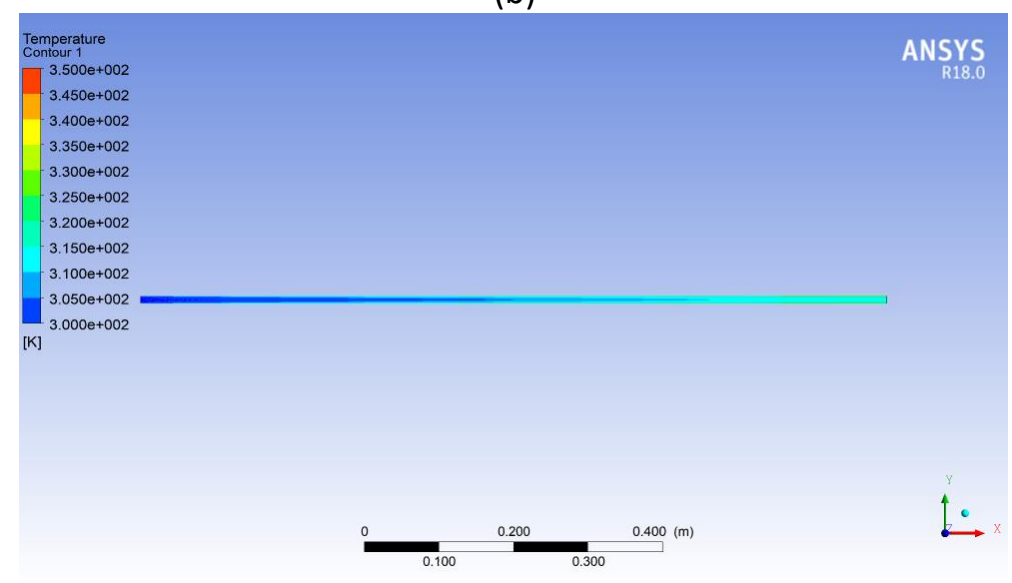

(c)

Fig. 9. Temperature contour for Re 40000 for particle concentration I $1 \%$, (b) $4 \%$ and (c) $6 \%$ for Mixture model

The reasons behind difference in performence with the variation in particle concentration lie in the principles of this two models. Whereas the VOF model solves only one set of momentum equation which is shared by both phases with respective densities, mixture model solves the mixture momentum equation where the mixture density and mixture viscocity are taken into account. Furthermore, the VOF model recognizes nanoparticles not as solids but as another much denser fluid which are spherical and have different properties and not immiscible with the base fluid. This is why VOF model takes surface tension between fluids into account which causes impact on heat transfer. As for the mixture model, it takes base fluid and nanoparticles as two different phases where particles 
are treated as solids and the model includes drag force and collisions in the calculation. In addition it considers the slip velocities between phases that the VOF model tends to ignore by which the prediction of heat trasnfer is varied. At lower particle concentration the presence and effect of nanoparticles on the properties and behaviour of nanofluid as a distinct phase is low and mostly at these conditions nanofluids behaviour tips on the side of fluid. But as concentration is increased the effect of nanoparticles in the fluid starts becoming much visible and apparent in terms of exerted forces, difference in properties and other related variables. So with the increase of particle concentration it becomes more important to treat these phases differently and to treat phase interactions accordingly that the mixture model does but the VOF model seems to disregard specially when one phase is solid.

One more point to be noted that, for lower concentration numerical simulation underestimates $\mathrm{Nu}$ whereas for higher concentrations it overestimates $\mathrm{Nu}$ and this is true for both models. This implies that, there must be a certain concentration between $1 \%$ and $4 \%$ which will yield the same result by simulation and experiment in terms of heat transfer enhancement $(\mathrm{Nu})$ and will contain $0 \%$ error. Further experimental work as well as simulation need to be conducted on this particle concentration range to find out such concentration that will yield $0 \%$ error with experimental data.

Some of the temperature contours for both models simulation are shown below in Figure 9 and Figure 10.

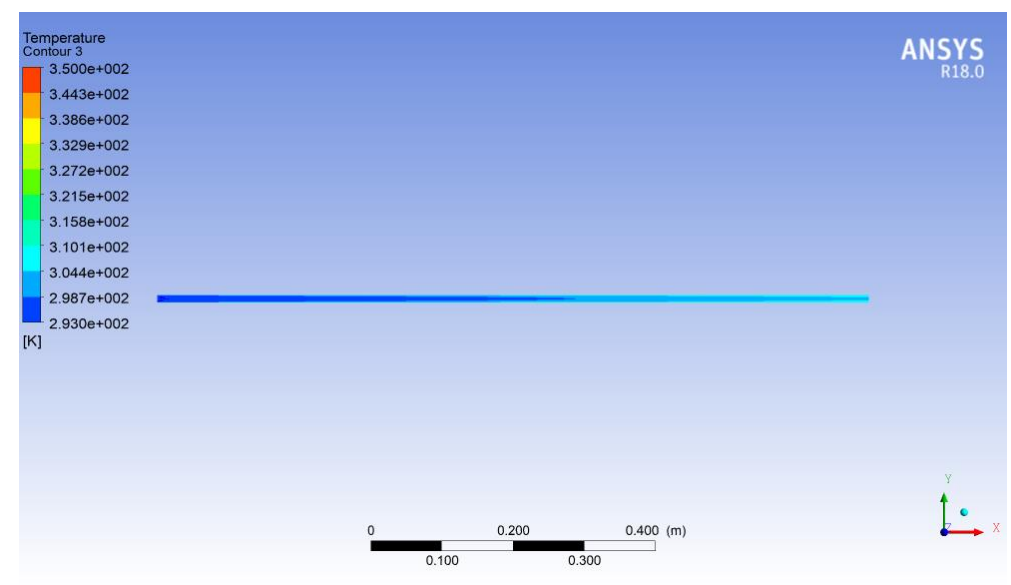

(a)

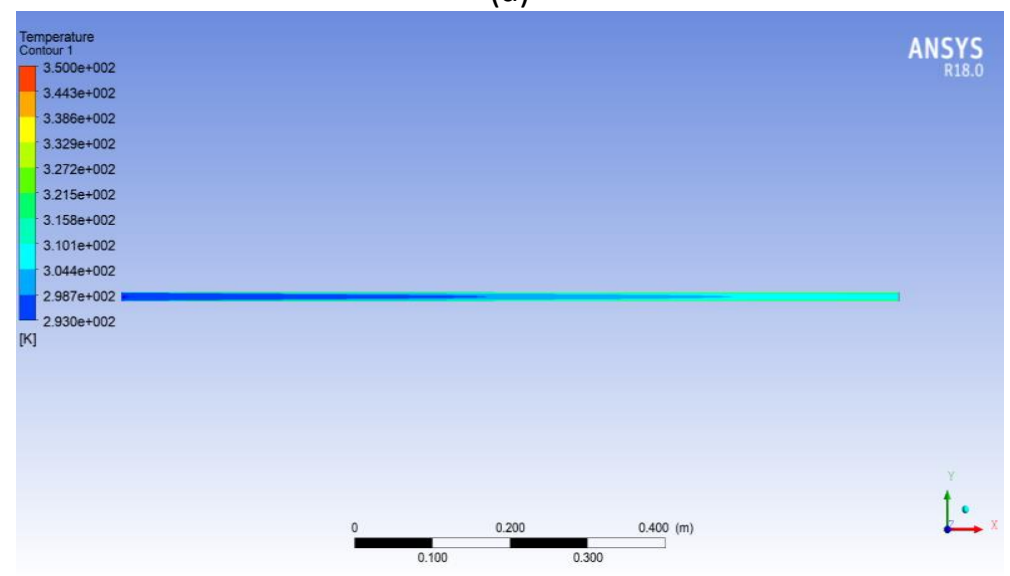

(b) 


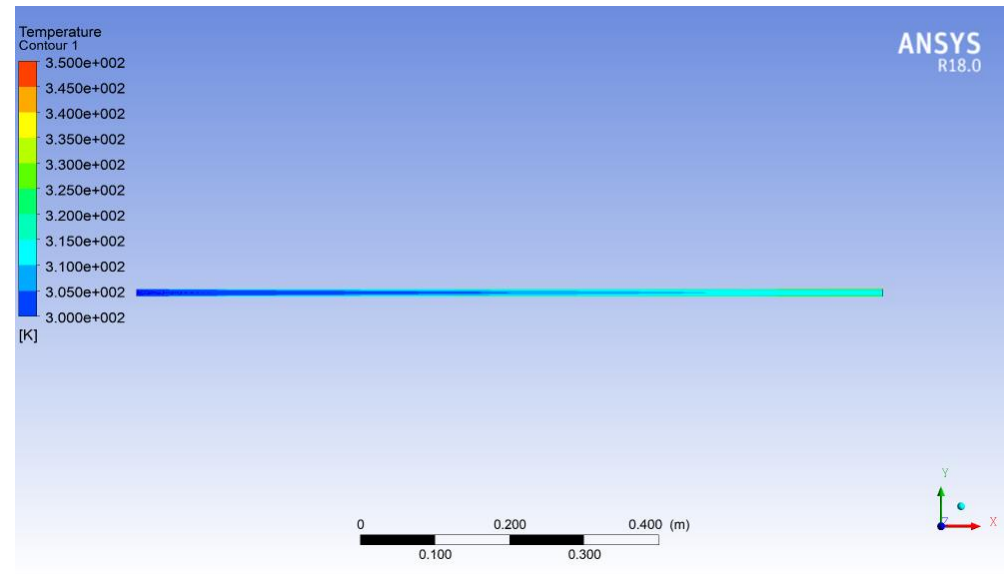

(c)

Fig. 10. Temperature contour for Re 40000 for particle concentration (a) $1 \%$, (b) $4 \%$ and (c) $6 \%$ for VOF model

\section{Conclusion}

Both models show good agreement with experimental data at intermediate concentration. As particle concentration gets higher the deviation from experimental data seems to be getting significant. This can be said that heat transfer of nanofluids increases with rise in particle concentration and Re. At lower concentration VOF model seems to predict heat transfer enhancement better but for higher concentrations Mixture model should be considered. Some further study can be conducted on the particle concentration range $1 \%$ to $4 \%$ to examine the heat transfer behavior of water- $\mathrm{Al}_{2} \mathrm{O}_{3}$ nanofluid more perfectly.

\section{Acknowledgement}

This research was not funded by any grant.

\section{References}

[1] Sreelakshmy, K. R., Aswathy S. Nair, K. Vidhya, T. Saranya, and Sreeja C. Nair. "An overview of recent nanofluid research." International Research Journal of Pharmacy 5, no. 4 (2014): 239-243. https://doi.org/10.7897/22308407.050451

[2] Lee, Ji-Hwan, Seung-Hyun Lee, Chul Choi, Seok Jang, and Stephen Choi. "A Review of Thermal Conductivity Data, Mechanisms and Models for Nanofluids." International Journal of Micro-Nano Scale Transport (2011). https://doi.org/10.1260/1759-3093.1.4.269

[3] Mousavi, S. M., F. Esmaeilzadeh, and X. P. Wang. "Effects of temperature and particles volume concentration on the thermophysical properties and the rheological behavior of $\mathrm{CuO} / \mathrm{MgO} / \mathrm{TiO} 2$ aqueous ternary hybrid nanofluid." Journal of Thermal Analysis and Calorimetry 137, no. 3 (2019): 879-901. https://doi.org/10.1007/s10973-01908006-0

[4] Kriby, Saliha, Mohamed Announ, and Tayeb Kermezli. "2D CFD simulation to investigate the thermal and hydrodynamic behavior of nanofluid flowing through a pipe in turbulent conditions." CFD Letters 11, no. 11 (2019): 58-75.

[5] Fard, M. Haghshenas, M. Nasr Esfahany, and M. R. Talaie. "Numerical study of convective heat transfer of nanofluids in a circular tube two-phase model versus single-phase model." International Communications in Heat and Mass Transfer 37, no. 1 (2010): 91-97. https://doi.org/10.1016/i.icheatmasstransfer.2009.08.003

[6] Saghir, M. Ziad, Amirhossein Ahadi, Tooraj Yousefi, and Bahram Farahbakhsh. "Two-phase and single phase models of flow of nanofluid in a square cavity: comparison with experimental results." International Journal of Thermal Sciences 100 (2016): 372-380. https://doi.org/10.1016/i.ijthermalsci.2015.10.005

[7] Esfandiary, M., A. Habibzadeh, and H. Sayehvand. "Numerical Study of Single Phase/Two-Phase Models for Nanofluid Forced Convection and Pressure Drop in a Turbulence Pipe Flow." Transp Phenom Nano Micro Scales 4, no. 1 (2016): 11-18. 
[8] Kakaç, Sadık, and Anchasa Pramuanjaroenkij. "Single-phase and two-phase treatments of convective heat transfer enhancement with nanofluids-A state-of-the-art review." International Journal of Thermal Sciences 100 (2016): $75-$ 97. https://doi.org/10.1016/i.ijthermalsci.2015.09.021

[9] Lotfi, R., Y. Saboohi, and A. M. Rashidi. "Numerical study of forced convective heat transfer of nanofluids: comparison of different approaches." International Communications in Heat and Mass Transfer 37, no. 1 (2010): 74-78. https://doi.org/10.1016/i.icheatmasstransfer.2009.07.013

[10] Akbari, M., N. Galanis, and A. Behzadmehr. "Comparative analysis of single and two-phase models for CFD studies of nanofluid heat transfer." International Journal of Thermal Sciences 50, no. 8 (2011): 1343-1354. https://doi.org/10.1016/j.ijthermalsci.2011.03.008

[11] Göktepe, Sinan, Kunt Atalık, and Hakan Ertürk. "Comparison of single and two-phase models for nanofluid convection at the entrance of a uniformly heated tube." International Journal of Thermal Sciences 80 (2014): 83 92. https://doi.org/10.1016/i.ijthermalsci.2014.01.014

[12] Safaei, Mohammad Reza, A. Jahanbin, Ali Kianifar, Samira Gharehkhani, Akeel Shebeeb Kherbeet, Marjan Goodarzi, and Mahidzal Dahari. "Mathematical modeling for nanofluids simulation: a review of the latest works." Modeling and Simulation in Engineering Sciences (2016): 189-220. https://doi.org/10.5772/64154

[13] Najim, Monssif, Abderrahman Nait Alla, and Adil Charef. "Comparative numerical study of single and two-phase models of nanofluid liquid film evaporation in a vertical channel." In MATEC Web of Conferences, vol. 307, p. 01034. EDP Sciences, 2020. https://doi.org/10.1051/matecconf/202030701034

[14] Ambreen, Tehmina, Arslan Saleem, and Cheol Woo Park. "Homogeneous and Multiphase Analysis of Nanofluids Containing Nonspherical MWCNT and GNP Nanoparticles Considering the Influence of Interfacial Layering." Nanomaterials 11, no. 2 (2021): 277. https://doi.org/10.3390/nano11020277

[15] Hanafizadeh, P., M. Ashjaee, M. Goharkhah, K. Montazeri, and M. Akram. "The comparative study of single and two-phase models for magnetite nanofluid forced convection in a tube." International Communications in Heat and Mass Transfer 65 (2015): 58-70. https://doi.org/10.1016/i.icheatmasstransfer.2015.04.012

[16] Davarnejad, Reza, and Maryam Jamshidzadeh. "CFD modeling of heat transfer performance of MgO-water nanofluid under turbulent flow." Engineering Science and Technology, an International Journal 18, no. 4 (2015): 536-542. https://doi.org/10.1016/i.jestch.2015.03.011

[17] Bianco, Vincenzo, Oronzio Manca, and Sergio Nardini. "Numerical simulation of water/AI2O3 nanofluid turbulent convection." Advances in Mechanical Engineering 2 (2010): 976254. https://doi.org/10.1155/2010/976254

[18] Launder, B. E., and D. B. Spalding. "The numerical computation of turbulent flows." Computer Methods in Applied Mechanics and Engineering 3, no. 2 (1974): 269-289. https://doi.org/10.1016/0045-7825(74)90029-2

[19] Alawadhi, Esam M. Finite element simulations using ANSYS. CRC Press, 2015. https://doi.org/10.1201/b18949

[20] Pak, Bock Choon, and Young I. Cho. "Hydrodynamic and heat transfer study of dispersed fluids with submicron metallic oxide particles." Experimental Heat Transfer an International Journal 11, no. 2 (1998): 151-170. https://doi.org/10.1080/08916159808946559 